\title{
Exact master equations for the non-Markovian decay of a qubit
}

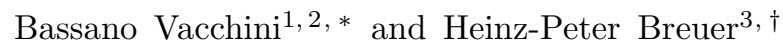 \\ ${ }^{1}$ Università degli Studi di Milano, Dipartimento di Fisica, Via Celoria 16, I-20133 Milano, Italy \\ ${ }^{2}$ INFN Sezione di Milano, Via Celoria 16, I-20133 Milano, Italy \\ ${ }^{3}$ Physikalisches Institut, Universität Freiburg, Hermann-Herder-Strasse 3, D-79104 Freiburg, Germany
}

(Dated: October 24, 2018)

\begin{abstract}
Exact master equations describing the decay of a two-state system into a structured reservoir are constructed. Employing the exact solution for the model we determine analytical expressions for the memory kernel of the Nakajima-Zwanzig master equation and for the generator of the corresponding time-convolutionless master equation. This approach allows a detailed investigation and comparison of the convergence behavior of the corresponding perturbation expansions. Moreover, we find that the structure of widely used phenomenological master equations with memory kernel may be incompatible with a non-perturbative treatment of the underlying microscopic model. We discuss several physical implications of our results on the microscopic analysis and the phenomenological modelling of non-Markovian quantum dynamics of open systems.
\end{abstract}

PACS numbers: 03.65.Yz,03.65.Ta,42.50.Lc

\section{INTRODUCTION}

The field of open quantum systems [1-3] is of great interest because of its relevance in applications of quantum mechanics, as well as in a deeper understanding of the theory itself. Indeed the study of the interaction between a quantum system and its environment is an endeavor common to many fields such as quantum measurement theory, quantum communication, quantum optics, condensed matter theory and quantum chemistry to name a few. The field is well assessed as far as Markovian dynamics is concerned, in which the Gorini-KossakowskiSudarshan-Lindblad expression for the generator of a quantum dynamical semigroup [4, 5] provides a benchmark result for both microscopic and phenomenological approaches. This situation is however not satisfactory when one has to go beyond the Born-Markov approximation and considers systems in which a separation of time scales between system and environment can no longer be assumed in a realistic description. Memory effects then become important and a non-Markovian description is mandatory. For this case a general consistent theoretical framework has not yet been found, and partial results have been obtained as a result of intense efforts (see, e.g., Refs. 6 [21]). An important step in the development of a general theory consists in the construction of a suitable measure that quantifies the degree of non-Markovianity for a given dynamical evolution 22, 23].

In this article we will obtain the exact NakajimaZwanzig kernel for a two-level system coupled to a Bosonic reservoir discussed in [2], and compare it to the exact time-convolutionless master equation as well as to the Markovian approximation of the dynamics. This will show how involved the transition from the approx-

\footnotetext{
*Electronic address: vacchini@mi.infn.it

${ }^{\dagger}$ Electronic address: breuer@physik.uni-freiburg.de
}

imate Markovian level of description to the exact nonMarkovian regime can be. Indeed, the non-Markovian memory kernel is found to have an operator structure which differs from the one that appears in the BornMarkov approximation. Often one tries to obtain dynamical equations of motion for non-Markovian systems by slight modifications with respect to the Markovian case, e.g., by considering a master equation which involves a superoperator given by a convolution in time of the corresponding Markovian superoperator [24 29]. Our results show that such an approach, although being justified as a phenomenological modelling, can be incompatible with a non-perturbative treatment of the underlying microscopic system-environment model. Moreover, different perturbation expansions such as time-convolutionless and Nakajima-Zwanzig projection operator technique turn out to have different ranges of validity. Indeed the timeconvolutionless expansion breaks down at finite time in the strong coupling limit, while the Nakajima-Zwanzig approach does not preserve positivity if restricted to second order. Furthermore the convergence to the exact solution is not uniform with respect to the expansion parameter: Different matrix elements of the statistical operator such as coherences and populations are obtained with quite different accuracy at the same perturbative order.

The paper is organized as follows. In Sec. III we introduce the model and its exact solution, which is later exploited to obtain the exact equations of motion for the reduced statistical operator of the system. In Sec. III we recall the structure of the time-convolutionless master equation, pointing out two different perturbation expansions for the generator. In Sec. [V] we derive the Nakajima-Zwanzig integral kernel, providing an alternative expansion with respect to the standard method. The two results are compared in Sec. $\mathrm{V}$, also building on an exact analytic expression for all the quantities involved obtained considering a Lorentzian spectral density. We finally draw our conclusions in Sec. VI. 


\section{THE MODEL AND ITS EXACT SOLUTION}

The total Hamiltonian of the model is given by

$$
H=H_{S}+H_{E}+H_{I}=H_{0}+H_{I},
$$

where

$$
H_{S}=\omega_{0} \sigma_{+} \sigma_{-}
$$

describes a two-state system (qubit) with ground state $|0\rangle$, excited state $|1\rangle$ and transition frequency $\omega_{0}$. The operators $\sigma_{+}=|1\rangle\langle 0|$ and $\sigma_{-}=|0\rangle\langle 1|$ are the raising and lowering operators of the qubit. The environmental Hamiltonian is taken to be

$$
H_{E}=\sum_{k} \omega_{k} b_{k}^{\dagger} b_{k}
$$

describing a collection of harmonic oscillators with creation and annihilation operators $b_{k}^{\dagger}$ and $b_{k}$ which satisfy bosonic commutation relations $\left[b_{k}, b_{k^{\prime}}^{\dagger}\right]=\delta_{k k^{\prime}}$. The interaction Hamiltonian takes the form

$$
H_{I}=\sum_{k}\left(g_{k} \sigma_{+} \otimes b_{k}+g_{k}^{*} \sigma_{-} \otimes b_{k}^{\dagger}\right) .
$$

The model thus describes for example the coupling of the qubit to a reservoir of electromagnetic field modes labelled by the index $k$ with corresponding frequencies $\omega_{k}$ and coupling constants $g_{k}$, and has already been discussed in [2].

In the following we will work in the interaction picture with respect to $H_{0}=H_{S}+H_{E}$. In this picture the Schrödinger equation reads

$$
\frac{d}{d t}|\Psi(t)\rangle=-i H_{I}(t)|\Psi(t)\rangle
$$

where the interaction Hamiltonian is given by

$$
H_{I}(t)=\sigma_{+}(t) \otimes B(t)+\sigma_{-}(t) \otimes B^{\dagger}(t)
$$

with

$$
\sigma_{ \pm}(t)=\sigma_{ \pm} e^{ \pm i \omega_{0} t}
$$

and

$$
B(t)=\sum_{k} g_{k} b_{k} e^{-i \omega_{k} t} .
$$

It is easy to verify that the operator

$$
N=\sigma_{+} \sigma_{-}+\sum_{k} b_{k}^{\dagger} b_{k}
$$

for the number of excitations in the system commutes both with the total Hamiltonian $H$ and with the interaction Hamiltonian $H_{I}(t)$, which is a consequence of the fact that the rotating wave approximation has been used in the interaction Hamiltonian (4). It follows that any initial state of the form

$$
\begin{aligned}
|\Psi(0)\rangle= & c_{0}|0\rangle \otimes|0\rangle_{E}+c_{1}(0)|1\rangle \otimes|0\rangle_{E} \\
& +\sum_{k} c_{k}(0)|0\rangle \otimes|k\rangle_{E}
\end{aligned}
$$

evolves after time $t$ into the state

$$
\begin{aligned}
|\Psi(t)\rangle= & c_{0}|0\rangle \otimes|0\rangle_{E}+c_{1}(t)|1\rangle \otimes|0\rangle_{E} \\
& +\sum_{k} c_{k}(t)|0\rangle \otimes|k\rangle_{E} .
\end{aligned}
$$

The state $|0\rangle_{E}$ denotes the vacuum state of the reservoir, and $|k\rangle_{E}=b_{k}^{\dagger}|0\rangle_{E}$ the state with one particle in mode $k$. Note that the amplitudes $c_{1}(t)$ and $c_{k}(t)$ depend on time, while the amplitude $c_{0}$ is constant in time because of $H_{I}(t)|0\rangle \otimes|0\rangle_{E}=0$. Substituting Eq. (11) into the Schrödinger equation (5) one finds

$$
\begin{aligned}
\frac{d}{d t} c_{1}(t) & =-i \sum_{k} g_{k} e^{i\left(\omega_{0}-\omega_{k}\right) t} c_{k}(t), \\
\frac{d}{d t} c_{k}(t) & =-i g_{k}^{*} e^{-i\left(\omega_{0}-\omega_{k}\right) t} c_{1}(t) .
\end{aligned}
$$

We assume in the following that $c_{k}(0)=0$. This means that the environment is in the vacuum state initially and that the total initial state is given by the product state

$$
|\Psi(0)\rangle=\left(c_{0}|0\rangle+c_{1}(0)|1\rangle\right) \otimes|0\rangle_{E} \equiv|\psi(0)\rangle \otimes|0\rangle_{E}
$$

Expressing $c_{k}(t)$ in terms of $c_{1}(t)$ by means of Eq. (13) and substituting the result into Eq. (12) one obtains an integrodifferential equation for the amplitude $c_{1}(t)$,

$$
\frac{d}{d t} c_{1}(t)=-\int_{0}^{t} d t_{1} f\left(t-t_{1}\right) c_{1}\left(t_{1}\right) .
$$

Given the solution of this equation, which can be found through a Laplace transformation, the amplitudes $c_{k}(t)$ are determined by Eq. (13). The kernel $f\left(t-t_{1}\right)$ of Eq. (15) is given by a certain two-point correlation function of the reservoir,

$$
\begin{aligned}
f\left(t-t_{1}\right) & =\left\langle 0\left|B(t) B^{\dagger}\left(t_{1}\right)\right| 0\right\rangle e^{i \omega_{0}\left(t-t_{1}\right)} \\
& =\sum_{k}\left|g_{k}\right|^{2} e^{i\left(\omega_{0}-\omega_{k}\right)\left(t-t_{1}\right)}
\end{aligned}
$$

on which no restrictive hypothesis is made, so that our results will be valid for an environment with a generic spectral density.

With the help of the procedure described above, already used by Weisskopf and Wigner in their classical paper [30], one finds the solution of the Schrödinger equation of the total system with initial states of the form (14) lying in the sector of the Hilbert space corresponding to zero or one excitations. By means of this solution we can construct the exact dynamical map describing the 
time-evolution of the reduced density matrix of the qubit which is given by

$$
\rho(t)=\operatorname{tr}_{E}\{|\Psi(t)\rangle\langle\Psi(t)|\}=\left(\begin{array}{cc}
\rho_{11}(t) & \rho_{10}(t) \\
\rho_{01}(t) & \rho_{00}(t)
\end{array}\right),
$$

where $\rho_{i j}(t)=\langle i|\rho(t)| j\rangle$ for $i, j=0,1$. Using Eq. (11) we find

$$
\begin{aligned}
& \rho_{11}(t)=1-\rho_{00}(t)=\left|c_{1}(t)\right|^{2}, \\
& \rho_{10}(t)=\rho_{01}^{*}(t)=c_{0}^{*} c_{1}(t) .
\end{aligned}
$$

It is convenient to define the function $G(t)$ as the solution of the equation

$$
\frac{d}{d t} G(t)=-\int_{0}^{t} d t_{1} f\left(t-t_{1}\right) G\left(t_{1}\right)
$$

corresponding to the initial condition $G(0)=1$. We then have $c_{1}(t)=G(t) c_{1}(0)$ and, hence, the dynamics of the elements of the reduced density matrix can be represented as follows,

$$
\begin{aligned}
& \rho_{11}(t)=|G(t)|^{2} \rho_{11}(0) \\
& \rho_{00}(t)=\rho_{00}(0)+\left(1-|G(t)|^{2}\right) \rho_{11}(0), \\
& \rho_{10}(t)=G(t) \rho_{10}(0) \\
& \rho_{01}(t)=G^{*}(t) \rho_{01}(0)
\end{aligned}
$$

These equations have been derived for the pure product initial state (14), i.e., they describe the time-evolution corresponding to the pure reduced system's initial state $\rho(0)=|\psi(0)\rangle\langle\psi(0)|$. However, since any mixed initial state can be represented as convex-linear combination of pure initial states, and since the function $G(t)$ introduced above does not depend on the initial condition, the equations (21)-(24) hold true for any pure or mixed initial state. They thus represent the exact dynamical map $\Phi(t)$ which transforms the initial states into the states at time $t$ :

$$
\Phi(t): \rho(0) \mapsto \rho(t)=\Phi(t) \rho(0), \quad t \geq 0 .
$$

Since we have constructed this map from the exact solution of the model, it is clear from the general theory of open quantum systems that $\Phi(t)$ is completely positive and trace preserving.

\section{THE TIME-CONVOLUTIONLESS MASTER EQUATION}

\section{A. Exact master equation in time-convolutionless form}

The exact solution determined in Sec. II enables the construction of the exact generator $\mathcal{K}_{\text {TCL }}$ of the timeconvolutionless master equation

$$
\frac{d}{d t} \rho(t)=\mathcal{K}_{\mathrm{TCL}}(t) \rho(t)
$$

governing the dynamics of the reduced density matrix. The time-convolutionless generator is defined in terms of the dynamical map $\Phi(t)$ by means of

$$
\mathcal{K}_{\mathrm{TCL}}(t)=\dot{\Phi}(t) \Phi^{-1}(t)
$$

provided the inverse map $\Phi^{-1}(t)$ exists. Using then Eqs. (21)-(24) one shows that the generator takes the following form [2],

$$
\begin{aligned}
\mathcal{K}_{\mathrm{TCL}}(t) \rho= & -\frac{i}{2} S(t)\left[\sigma_{+} \sigma_{-}, \rho\right] \\
& +\gamma(t)\left[\sigma_{-} \rho \sigma_{+}-\frac{1}{2}\left\{\sigma_{+} \sigma_{-}, \rho\right\}\right],
\end{aligned}
$$

where we have introduced the definitions

$$
\gamma(t)=-2 \Re\left(\frac{\dot{G}(t)}{G(t)}\right), \quad S(t)=-2 \Im\left(\frac{\dot{G}(t)}{G(t)}\right) .
$$

By construction, Eq. (26) with the generator (28) represents an exact time-local master equation. Note that the generator is well-defined as long as $G(t) \neq 0$. The quantity $S(t)$ plays the role of a time-dependent frequency shift, and $\gamma(t)$ can be interpreted as a time-dependent decay rate. We observe that the structure of $\mathcal{K}_{\mathrm{TCL}}$ is similar to that of a Lindblad generator. However, due to the time dependence of the coefficients $S(t)$ and $\gamma(t)$ Eq. (26) does generally not yield a quantum dynamical semigroup. Moreover, the time-dependent rate $\gamma(t)$ may become negative, signifying strong non-Markovian behavior of the reduced system dynamics.

\section{B. Perturbation expansions of the generator}

In most cases of interest the time-convolutionless generator can only be determined through a perturbation expansion. Here we investigate two methods of expanding the exact master equation (26) with respect to the strength of the interaction Hamiltonian $H_{I}$. To this end, we introduce a small overall expansion parameter $\alpha$, replacing the coupling constants $g_{k}$ in the interaction Hamiltonian (4) by $\alpha g_{k}$. The two-point correlation function $f(t)$, being proportional to $\alpha^{2}$, is then to be regarded as a quantity of second order.

The first method consists in using Eq. (20) to obtain a perturbative expression for $G(t)$ from which one directly finds an expansion for the coefficients $\gamma(t)$ and $S(t)$ appearing in the master equation. The expansion of $G(t)$ is obviously of the form

$$
G(t)=\sum_{n=0}^{\infty} \alpha^{2 n} G^{(2 n)}(t)
$$

where $G^{(0)}(t) \equiv 1$ because of the required initial condition $G(0)=1$, and Eq. (20) leads to the following recursion relation

$$
G^{(2 n)}(t)=-\int_{0}^{t} d t_{1} \int_{0}^{t_{1}} d t_{2} f\left(t_{1}-t_{2}\right) G^{(2 n-2)}\left(t_{2}\right) .
$$


To illustrate the procedure we determine the frequency shift and the decay rate to fourth order in $\alpha$ :

$$
\begin{aligned}
& -\frac{1}{2}[\gamma(t)+i S(t)]=\frac{\dot{G}(t)}{G(t)} \\
& \quad=\alpha^{2} \dot{G}^{(2)}(t)+\alpha^{4}\left[\dot{G}^{(4)}(t)-\dot{G}^{(2)}(t) G^{(2)}(t)\right]+\mathcal{O}\left(\alpha^{6}\right) .
\end{aligned}
$$

With the help of these expressions one obtains the second and the fourth order contributions for the coefficients of the master equation:

$$
\begin{aligned}
& \gamma^{(2)}(t)+i S^{(2)}(t)=2 \int_{0}^{t} d t_{1} f\left(t-t_{1}\right) \\
& \gamma^{(4)}(t)+i S^{(4)}(t)=2 \int_{0}^{t} d t_{1} \int_{0}^{t_{1}} d t_{2} \int_{0}^{t_{2}} d t_{3} \\
& \times\left[f\left(t-t_{2}\right) f\left(t_{1}-t_{3}\right)+f\left(t-t_{3}\right) f\left(t_{1}-t_{2}\right)\right]
\end{aligned}
$$

Another possibility for the construction of the perturbation expansion is to use the general method of expanding the time-convolutionless generator in terms of the ordered cumulants. This procedure allows to write a closed expression for the coefficients of the master equation which takes the form (for details, see [2] and references therein):

$$
\begin{gathered}
\gamma^{(2 n)}(t)+i S^{(2 n)}(t)=\int_{0}^{t} d t_{1} \int_{0}^{t_{1}} d t_{2} \ldots \int_{0}^{t_{2 n-2}} d t_{2 n-1}(34) \\
\times 2(-1)^{n+1}\left\langle f\left(t-t_{1}\right) f\left(t_{2}-t_{3}\right) \ldots f\left(t_{2 n-2}-t_{2 n-1}\right)\right\rangle_{\mathrm{oc}} .
\end{gathered}
$$

\section{THE NAKAJIMA-ZWANZIG MASTER EQUATION}

\section{A. The exact memory kernel}

The Nakajima-Zwanzig master equation is given by

$$
\frac{d}{d t} \rho(t)=\int_{0}^{t} d t_{1} \mathcal{K}_{\mathrm{NZ}}\left(t-t_{1}\right) \rho\left(t_{1}\right),
$$

where the superoperator $\mathcal{K}_{\mathrm{NZ}}(\tau)$ represents the memory kernel. We construct the form of this kernel from the exact solution of our model obtained in Sec. II. To this end, we employ the following Ansatz,

$$
\begin{aligned}
\mathcal{K}_{\mathrm{NZ}}(\tau) \rho= & -i \varepsilon(\tau)\left[\sigma_{+} \sigma_{-}, \rho\right] \\
& +k_{1}(\tau)\left[\sigma_{-} \rho \sigma_{+}-\frac{1}{2}\left\{\sigma_{+} \sigma_{-}, \rho\right\}\right] \\
& +k_{2}(\tau)\left[\sigma_{+} \sigma_{-} \rho \sigma_{+} \sigma_{-}-\frac{1}{2}\left\{\sigma_{+} \sigma_{-}, \rho\right\}\right]
\end{aligned}
$$

where the functions $\varepsilon(\tau), k_{1}(\tau)$ and $k_{2}(\tau)$ are real, such that the master equation preserves Hermiticity and trace. The equations of motion for the population $\rho_{11}(t)$ and the coherence $\rho_{10}(t)$ obtained from this master equation read:

$$
\frac{d}{d t} \rho_{11}(t)=-\int_{0}^{t} d t_{1} k_{1}\left(t-t_{1}\right) \rho_{11}\left(t_{1}\right)
$$

and

$$
\begin{aligned}
& \frac{d}{d t} \rho_{10}(t)=-\int_{0}^{t} d t_{1} \\
& \quad \times\left[\frac{1}{2}\left\{k_{1}\left(t-t_{1}\right)+k_{2}\left(t-t_{1}\right)\right\}+i \varepsilon\left(t-t_{1}\right)\right] \rho_{10}\left(t_{1}\right) .
\end{aligned}
$$

On the other hand, Eq. (23) together with Eq. (20) yields:

$$
\frac{d}{d t} \rho_{10}(t)=-\int_{0}^{t} d t_{1} f\left(t-t_{1}\right) \rho_{10}\left(t_{1}\right),
$$

where we have set the expansion parameter $\alpha$ equal to one and we only have to remember that $f(t)$ is a quantity of second order. Comparing Eqn. (39) with Eq. (38) we see that the expression within the square brackets of (38) must be equal to $f\left(t-t_{1}\right)$, i. e., we get the conditions:

$$
\begin{aligned}
\varepsilon(\tau) & =f_{2}(\tau), \\
k_{1}(\tau)+k_{2}(\tau) & =2 f_{1}(\tau),
\end{aligned}
$$

where $f_{1}(\tau)$ and $f_{2}(\tau)$ denote the real and the imaginary part of the correlation function:

$$
f(\tau)=f_{1}(\tau)+i f_{2}(\tau)
$$

In order for Eq. (37) to reproduce the correct solution (21) we have to choose $k_{1}(\tau)$ in such a way that the solution of the equation

$$
\frac{d}{d t} z(t)=-\int_{0}^{t} d t_{1} k_{1}\left(t-t_{1}\right) z\left(t_{1}\right), \quad z(0)=1,
$$

is given by

$$
z(t)=|G(t)|^{2}
$$

Formulated in Laplace space this means that

$$
\hat{k}_{1}(u)=\frac{1-u \hat{z}(u)}{\hat{z}(u)} .
$$

Since the superoperator (36) preserves the Hermiticity and the trace of the density matrix, Eqs. (21)-(24) follow directly from Eqs. (37) and (38). Thus, we find that Eq. (36) represents the exact memory kernel of the model for any given two-point correlation function. In fact, given $f(\tau)$, the functions $\varepsilon(\tau), k_{1}(\tau)$ and $k_{2}(\tau)$ are uniquely determined by Eqs. (40), (41) and (45). In view of this result the memory kernel (36) can now be written in the form

$$
\begin{aligned}
\mathcal{K}_{\mathrm{NZ}}(\tau) \rho= & -i f_{2}(\tau)\left[\sigma_{+} \sigma_{-}, \rho\right]-f_{1}(\tau)\left\{\sigma_{+} \sigma_{-}, \rho\right\} \\
& +k_{1}(\tau) \sigma_{-} \rho \sigma_{+} \\
& +\left[2 f_{1}(\tau)-k_{1}(\tau)\right] \sigma_{+} \sigma_{-} \rho \sigma_{+} \sigma_{-},
\end{aligned}
$$

which only involves the real and the imaginary part of the correlation function and the function $k_{1}(\tau)$ which has to be determined from Eq. (45). 
We note that the various coefficients in the memory kernel exhibit a very different convergence behavior. In fact, we see that the commutator and the anticommutator term in Eq. (46) come out exactly in second order in $\alpha$. It follows that the equation of motion for the coherence $\rho_{10}$ [see Eq. (39)] is already reproduced exactly within second order, while the exact representation of the equation for the population $\rho_{11}$ requires in general the inclusion of all orders of the expansion. This non-uniform convergence behavior of the elements of the density matrix has been observed also in other, more complicated models [31], and seems to be a typical feature of the perturbation expansion of the memory kernel.

As will be shown below the relations (20) together with (43)-(45) provide a direct perturbation approach to the determination of the functions appearing in the memory kernel Eq. (36), as an alternative to the standard Nakajima-Zwanzig perturbation expansion. Moreover, this set of equations allows in some cases to derive a closed analytical expression for the memory kernel.

\section{B. Perturbation expansions of the memory kernel}

Here we discuss two methods of expanding the exact memory kernel with respect to the strength of the interaction Hamiltonian $H_{I}$. The first expansion method relies on the expansion Eq. (30) for the solution of Eq. (20), which determines the dynamical map $\Phi(t)$ once the twopoint correlation function $f(t)$ of the model given by Eq. (16) is specified.

Indeed as shown in Sec IV A to obtain the memory kernel Eq. (36) we only need to know the function $k_{1}(t)$. A perturbative expression for the latter can be easily obtained relying on the expansion Eq. (30) for $G(t)$, noting that thanks to Eq. (43) the Laplace transform of $k_{1}(t)$ can be directly expressed through Eq. (45) by means of the Laplace transform of the function $z(t)=|G(t)|^{2}$. This procedure leads to the following expansion

$$
k_{1}(t)=\sum_{n=0}^{\infty} k_{1}^{(2 n)}(t),
$$

as described in detail in Appendix A, where the zero order contribution is immediately seen to be zero.

Here we consider for the sake of simplicity only the second order contribution, which is readily obtained. According to Eq. (31) together with the initial condition $G(0)=1$ the expression for $G(t)$ up to second order is given by

$$
G(t) \approx 1-\int_{0}^{t} d t_{1} \int_{0}^{t_{1}} d t_{2} f\left(t_{2}\right),
$$

so that in the same approximation, recalling that the two-point correlation function $f(t)$ is a quantity of second order, one has

$$
z(t) \approx 1-2 \int_{0}^{t} d t_{1} \int_{0}^{t_{1}} d t_{2} f_{1}\left(t_{2}\right)
$$

where according to Eq. (42) $f_{1}(t)$ denotes the real part of the correlation function. The Laplace transform of this quantity is now easily expressed in terms of the Laplace transform of the correlation function according to

$$
\hat{z}(u) \approx \frac{u-2 \hat{f}_{1}(u)}{u^{2}},
$$

and further exploiting Eq. (45) we find

$$
\hat{k}_{1}(u) \approx 2 \hat{f}_{1}(u)
$$

This immediately implies for the second-order contributions to the kernel (36)

$$
k_{1}^{(2)}(t)=2 f_{1}(\tau)
$$

and therefore due to Eq. (41)

$$
k_{2}^{(2)}(\tau)=0
$$

As shown in Appendix $\mathrm{A}$ the fourth-order contribution reads

$$
\begin{aligned}
k_{1}^{(4)}\left(t-t_{1}\right)=-2 \Re \int_{t_{1}}^{t} d t_{2} \int_{t_{1}}^{t_{2}} d t_{3}\left[f\left(t-t_{3}\right) f\left(t_{1}-t_{2}\right)\right. \\
\left.+f\left(t-t_{1}\right) f\left(t_{3}-t_{2}\right)\right],
\end{aligned}
$$

so that $k_{2}^{(4)}=-k_{1}^{(4)}$. Indeed Eq. (41) generally implies $k_{2}^{(2 n)}=-k_{1}^{(2 n)}$ for $n \geq 2$, therefore Eq. (47) also provides an expansion for $k_{2}(t)$.

The second expansion method is to employ the general Nakajima-Zwanzig projection operator technique [32, 33] in which the memory kernel is expressed in terms of the full propagator of the total system. The details of this method for our model are presented in Appendix B. where it is shown that the projection operator technique reproduces, as expected, the above results obtained by the direct expansion of the coefficients in the memory kernel.

\section{DISCUSSION}

\section{A. Comparison of the time-convolutionless and the Nakajima-Zwanzig master equation}

It is interesting to compare the time-convolutionless master equation Eq. (26) with the Nakajima-Zwanzig master equation Eq. (35). For the considered model the functions appearing in Eq. (28) and Eq. (36) are given by Eq. (29) and Eqs. (40)-(41) respectively.

Comparing Eqs. (28) and (36) we see that the superoperator structure of the memory kernel differs from that of the time-convolutionless generator. In fact, the memory kernel (36) contains the term proportional to $k_{2}(\tau)$ which involves the projection $\sigma_{+} \sigma_{-}=|1\rangle\langle 1|$ onto the excited state. Without such a term the equations (37) and (38) for the population and the coherence would be 
incompatible with the exact expressions (21) and (23). However, a term with this structure is missing in the time-convolutionless generator (28). A further remarkable point is the fact that in second order $k_{2}(\tau)=0$, according to Eqs. (41) and (52). This shows that the difference in the superoperator structure of the memory kernel and the time-convolutionless generator is visible only in higher orders of the perturbation expansion.

The above discussion leads to some conclusions which are important for the modelling of non-Markovian dynamics through phenomenological master equations. In the Markovian limit our model yields the following Lindblad generator $\mathcal{L}$ describing a quantum dynamical semigroup,

$$
\mathcal{L} \rho=-\frac{i}{2} S_{M}\left[\sigma_{+} \sigma_{-}, \rho\right]+\gamma_{M}\left[\sigma_{-} \rho \sigma_{+}-\frac{1}{2}\left\{\sigma_{+} \sigma_{-}, \rho\right\}\right]
$$

with constant frequency shift $S_{M}$ and decay rate $\gamma_{M} \geq 0$. Usually master equations of this form are derived by applying the Markov approximation and second order perturbation theory (Born-Markov approximation). A natural and widely-used non-Markovian generalization is then obtained from this equation by keeping the structure of the Lindblad generator $\mathcal{L}$ and by introducing a certain kernel function $h(\tau)$ to arrive at a master equation of the form $[24,28]$

$$
\frac{d}{d t} \rho(t)=\int_{0}^{t} d t_{1} h\left(t-t_{1}\right) \mathcal{L} \rho\left(t_{1}\right) .
$$

Although this equation is perfectly justified as a phenomenological ansatz, it does in general not represent the correct structure of the memory kernel of the underlying microscopic model. In fact, we see that even for the simple model studied here the true memory kernel (36) is not of the form of Eq. (56), but involves additional terms that are absent in the Markovian Lindblad generator. Indeed it is rather given by a linear combination of terms of the form Eq. (56), where going beyond the Born approximation besides the Markovian Lindblad generator other operator structures appear, which are still in Lindblad form but with different Lindblad operators. This observation seems to be of particular relevance for the analysis of the positivity and the complete positivity of the dynamical maps obtained from phenomenological equations of motion.

\section{B. Example}

These considerations can be nicely illustrated considering the example of an exponential correlation function, corresponding to a Lorentzian spectral density [2]

$$
f(\tau)=\frac{1}{2} \gamma_{0} \lambda e^{-\lambda|\tau|},
$$

where the parameters $\gamma_{0}$ and $\lambda$ are real and positive. For this case both time-convolutionless generator and
Nakajima-Zwanzig kernel can be exactly calculated. Indeed by means of Eq. (20) one obtains for the function $G(t)$ the expression:

$$
G(t)=e^{-\lambda t / 2}\left[\cosh \left(\frac{\lambda t}{2} \delta\right)+\frac{1}{\delta} \sinh \left(\frac{\lambda t}{2} \delta\right)\right],
$$

where $\delta=\sqrt{1-2 \gamma_{0} / \lambda}$. Note that this function is always real. Furthermore it stays positive for any time $t$ in the weak coupling regime $\gamma_{0}<\lambda / 2$, while for strong coupling $\gamma_{0}>\lambda / 2$ the parameter $\delta$ becomes purely imaginary and the function $G(t)$ starts to oscillate. In particular it goes through zero for the first time when $t$ is equal to the smallest positive solution of

$$
t=\frac{2}{\lambda \hat{\delta}}(n \pi-\arctan \hat{\delta}),
$$

where $\hat{\delta}=\sqrt{2 \gamma_{0} / \lambda-1}$ and $n \in \mathbb{N}$. Building on Eq. (58) one can obtain the exact expressions for the functions $\gamma(t)$ and $S(t)$ appearing in the time-convolutionless generator, given by $S(t)=0$ and

$$
\gamma(t)=2 \gamma_{0} \frac{\sinh \left(\frac{\lambda t}{2} \delta\right)}{\delta \cosh \left(\frac{\lambda t}{2} \delta\right)+\sinh \left(\frac{\lambda t}{2} \delta\right)} .
$$

In order to obtain the Nakajima-Zwanzig kernel one considers the Laplace transform of the function $z(t)=$ $|G(t)|^{2}$ which is found to be:

$$
\hat{z}(u)=\frac{(u+\lambda)(u+2 \lambda)+\gamma_{0} \lambda}{(u+\lambda)\left[(u+\lambda)^{2}-\lambda^{2}+2 \gamma_{0} \lambda\right]},
$$

so that according to Eq. (45) one has:

$$
\hat{k_{1}}(u)=\gamma_{0} \lambda \frac{u+2 \lambda}{(u+\lambda)(u+2 \lambda)+\gamma_{0} \lambda} .
$$

Transforming back to the time domain we finally get

$$
k_{1}(t)=\gamma_{0} \lambda e^{-3 \lambda t / 2}\left[\cosh \left(\frac{\lambda t}{2} \delta^{\prime}\right)+\frac{1}{\delta^{\prime}} \sinh \left(\frac{\lambda t}{2} \delta^{\prime}\right)\right],
$$

where $\delta^{\prime}=\sqrt{1-4 \gamma_{0} / \lambda}$. Substituting this result into Eq. (46) we find the exact memory kernel for the case of an exponential correlation function.

The exact expressions Eq. (60) and Eq. (63) already allow for an important comparison. While the function on the right-hand side of Eq. (63) represents an analytic function of $\gamma_{0}$ (remember that $\gamma_{0}$ is a quantity of second order in the expansion parameter $\alpha$ ), so that the Nakajima-Zwanzig memory kernel has an infinite radius of convergence, the same does not hold true for the time-convolutionless generator. Indeed the timeconvolutionless expansion breaks down in the strong coupling regime $\gamma_{0}>\lambda / 2$ when the function $G(t)$ given in Eq. (58) goes through zero, corresponding to the divergence of the decay rate $\gamma(t)$ given in Eq. (60) and obtained from the relation Eq. (29). 
Considering an expansion in $\gamma_{0}$ of the function $k_{1}(t)$ given by Eq. (63) which fixes the memory kernel, due to the fact that the correlation function Eq. (57) is real one obtains

$$
\mathcal{K}_{\mathrm{NZ}}^{(2)}(\tau) \rho=2 f(\tau)\left[\sigma_{-} \rho \sigma_{+}-\frac{1}{2}\left\{\sigma_{+} \sigma_{-}, \rho\right\}\right],
$$

so that up to second-order the corresponding master equation is indeed of the form of Eq. (56) with the exponential kernel function $h(t)=2 f(t)$. However, in fourth order further terms appear which are not present in (56):

$$
\mathcal{K}_{\mathrm{NZ}}^{(4)}(\tau) \rho=k_{1}^{(4)}(\tau)\left[\sigma_{-} \rho \sigma_{+}-\sigma_{+} \sigma_{-} \rho \sigma_{+} \sigma_{-}\right],
$$

where

$$
k_{1}^{(4)}(\tau)=\gamma_{0}^{2}\left[e^{-\lambda \tau}(1-\lambda \tau)-e^{-2 \lambda \tau}\right] .
$$

As shown in 9] this implies in particular that if one truncates the expansion to first order in $\gamma_{0}$ the complete positivity (and even the positivity) of the resulting dynamical map is violated for strong couplings in the NakajimaZwanzig case. On the contrary the second-order timeconvolutionless master equation always guarantees complete positivity, as can be seen considering the secondorder approximation for Eq. (60) given by

$$
\gamma^{(2)}(t)=\gamma_{0}\left(1-e^{-\lambda t}\right)
$$

\section{CONCLUSIONS}

We have constructed the exact Nakajima-Zwanzig memory kernel for a specific model describing the decay of a two-level system into a reservoir of field modes which is initially in the vacuum state. The construction of the memory kernel is based on the analytical solution of the Schrödinger equation within the Hilbert space sector describing states with zero or one excitation, and is valid for a generic spectral density. Since the dynamical map giving the reduced system dynamics of the two-state system is known exactly, there is of course in principle no reason to resort to any kind of master equation in order to determine the dynamical behavior of the system. However, the present results lead to several important implications which are relevant for more realistic physical systems and their microscopic or phenomenological modelling, where analytical results cannot be obtained. Indeed for this model both time-convolutionless generator and Nakajima-Zwanzig kernel can be exactly expressed in terms of functions for which perturbative expansions are given, together with the exact solution for a reservoir with an exponential correlation function, corresponding to a Lorentzian spectral density. This allows for a detailed comparison of the two approaches expressing the dynamics in terms of a time-local and integrodifferential master equation respectively. It turns out that contrary to what is often expected the Nakajima-Zwanzig master equation is not simply obtained by convolution of the Lindblad operator appearing in the non-Markovian case with a suitable kernel. It actually has a different operator structure, emerging when considering higher perturbative orders. Furthermore the exact analytical result obtained for a Lorentzian spectral density shows the different convergence behavior of the two approaches. While the Nakajima-Zwanzig kernel is an analytic function of the coupling strength, providing a well-defined master equation at any time, the time-convolutionless generator breaks down at finite time in the strong coupling regime, thus failing to reproduce the asymptotic behavior.

\section{Acknowledgments}

This work was partially supported by MIUR under PRIN2008.

\section{Appendix A}

In this Appendix we shall consider how to obtain a perturbative expansion for the function $k_{1}(\tau)$ which according to Eqs. (36) and (40)-(41) determines the memory kernel in the Nakajima-Zwanzig master equation, as a function of the two-point correlation function $f(t)$ of the reservoir. To this end one considers the solution of Eq. (20) which is of the form Eq. (30) with $G(0)=1$ and $G^{(2 n)}(t)$ explicitly given by

$$
G^{(2 n)}(t)=(-)^{n} \int_{0}^{t} d t_{1} \int_{0}^{t_{1}} d t_{2} \cdots \int_{0}^{t_{2 n-1}} d t_{2 n} \prod_{i=1}^{n} f\left(t_{2 i-1}-t_{2 i}\right)
$$


so that

$$
\begin{aligned}
z(t)=|G(t)|^{2}= & 1+2 \Re \sum_{n=1}^{\infty}(-)^{n} \int_{0}^{t} d t_{1} \int_{0}^{t_{1}} d t_{2} \cdots \int_{0}^{t_{2 n-1}} d t_{2 n} \prod_{i=1}^{n} f\left(t_{2 i-1}-t_{2 i}\right) \\
& +\left|\sum_{n=1}^{\infty}(-)^{n} \int_{0}^{t} d t_{1} \int_{0}^{t_{1}} d t_{2} \cdots \int_{0}^{t_{2 n-1}} d t_{2 n} \prod_{i=1}^{n} f\left(t_{2 i-1}-t_{2 i}\right)\right|^{2} .
\end{aligned}
$$

Considering terms up to fourth order in the expansion parameter one has

$$
\begin{aligned}
z(t)= & 1-2 \Re \int_{0}^{t} d t_{1} \int_{0}^{t_{1}} d t_{2} f\left(t_{2}\right) \\
& +2 \Re \int_{0}^{t} d t_{1} \int_{0}^{t_{1}} d t_{2} \int_{0}^{t_{2}} d t_{3} \int_{0}^{t_{4}} d t_{4} f\left(t_{1}-t_{2}\right) f\left(t_{3}-t_{4}\right) \\
& +\left|\int_{0}^{t} d t_{1} \int_{0}^{t_{1}} d t_{2} f\left(t_{2}\right)\right|^{2}+\ldots
\end{aligned}
$$

and denoting real and imaginary parts of $f(t)$ as in Eq. (42) also

$$
\begin{aligned}
z(t)= & 1-2 \int_{0}^{t} d t_{1} \int_{0}^{t_{1}} d t_{2} f_{1}\left(t_{2}\right) \\
& +2 \int_{0}^{t} d t_{1} \int_{0}^{t_{1}} d t_{2} \int_{0}^{t_{2}} d t_{3} \int_{0}^{t_{3}} d t_{4}\left[f_{1}\left(t_{1}-t_{2}\right) f_{1}\left(t_{3}-t_{4}\right)-f_{2}\left(t_{1}-t_{2}\right) f_{2}\left(t_{3}-t_{4}\right)\right] \\
& +\left|\int_{0}^{t} d t_{1} \int_{0}^{t_{1}} d t_{2} f_{1}\left(t_{2}\right)\right|^{2}+\left|\int_{0}^{t} d t_{1} \int_{0}^{t_{1}} d t_{2} f_{2}\left(t_{2}\right)\right|^{2}+\ldots
\end{aligned}
$$

Introducing the functions

$$
h_{i}(t)=\int_{0}^{t} d t_{1} \int_{0}^{t_{1}} d t_{2} f_{i}\left(t_{2}\right) \quad i=1,2
$$

one obtains for the Laplace transform of $z(t)$

$$
\hat{z}(u)=\frac{u-2 u \widehat{f}_{1}(u)}{u^{2}}+\frac{2}{u^{3}}\left(\widehat{f}_{1}^{2}(u)-\widehat{f}_{2}^{2}(u)\right)+\widehat{h_{1}^{2}}(u)+\widehat{h_{2}^{2}}(u)+\ldots,
$$

and thanks to Eq. (45)

$$
\hat{k}_{1}(u)=2 \widehat{f}_{1}(u)+\frac{2}{u}\left(\widehat{f}_{1}^{2}(u)+{\widehat{f_{2}}}^{2}(u)\right)-u^{2}\left(\widehat{h_{1}^{2}}(u)+\widehat{h_{2}^{2}}(u)\right)+\ldots
$$

Using now the fact that the functions $h_{i}$ are equal to zero together with their derivatives at $t=0$ so that

$$
\widehat{\frac{\mathrm{d}^{2}}{d t^{2}} h_{i}^{2}}(u)=u^{2} \widehat{h_{i}^{2}}(u)
$$

one has

$$
\begin{aligned}
k_{1}(\tau)= & 2 f_{1}(\tau)+2 \int_{0}^{\tau} d t_{1} \int_{0}^{t_{1}} d t_{2} f_{1}\left(t_{1}-t_{2}\right) f_{1}\left(t_{2}\right)+2 \int_{0}^{\tau} d t_{1} \int_{0}^{t_{1}} d t_{2} f_{2}\left(t_{1}-t_{2}\right) f_{2}\left(t_{2}\right) \\
& -2\left|\int_{0}^{\tau} d t_{1} f_{1}\left(t_{1}\right)\right|^{2}-2\left|\int_{0}^{\tau} d t_{1} f_{2}\left(t_{1}\right)\right|^{2} \\
& -2 f_{1}(\tau) \int_{0}^{\tau} d t_{1} \int_{0}^{t_{1}} d t_{2} f_{1}\left(t_{1}-t_{2}\right)-2 f_{2}(\tau) \int_{0}^{\tau} d t_{1} \int_{0}^{t_{1}} d t_{2} f_{2}\left(t_{1}-t_{2}\right)+\ldots
\end{aligned}
$$

We now exploit the identity

$$
\int_{0}^{\tau} d t_{2} \int_{0}^{t_{2}} d t_{3} f\left(t_{2}-t_{3}\right) f\left(t_{3}\right)-\left|\int_{0}^{\tau} d t_{2} f_{1}\left(t_{2}\right)\right|^{2}+\int_{0}^{\tau} d t_{2} \int_{0}^{t_{2}} d t_{3} f\left(\tau-t_{3}\right) f\left(t_{2}\right)=0
$$


which can be checked noting that the function of $t$ defined by the left-hand side of Eq. (A10) has vanishing derivative and is equal to zero for $t=0$. We are thus left with

$$
\begin{aligned}
k_{1}(\tau)= & 2 f_{1}(\tau)-2 \int_{0}^{\tau} d t_{2} \int_{0}^{t_{2}} d t_{3}\left[f_{1}\left(\tau-t_{3}\right) f_{1}\left(t_{2}\right)+f_{1}(\tau) f_{1}\left(t_{2}-t_{3}\right)\right] \\
& -2 \int_{0}^{\tau} d t_{2} \int_{0}^{t_{2}} d t_{3}\left[f_{2}\left(\tau-t_{3}\right) f_{2}\left(t_{2}\right)+f_{2}(\tau) f_{2}\left(t_{2}-t_{3}\right)\right]+\ldots
\end{aligned}
$$

and thanks to the fact that real and imaginary parts of $f(t)$ are even and odd respectively

$$
\begin{aligned}
k_{1}(\tau)= & 2 f_{1}(\tau)-2 \int_{0}^{\tau} d t_{2} \int_{0}^{t_{2}} d t_{3}\left[f_{1}\left(\tau-t_{3}\right) f_{1}\left(-t_{2}\right)-f_{2}\left(\tau-t_{3}\right) f_{2}\left(-t_{2}\right)\right. \\
& \left.+f_{1}(\tau) f_{1}\left(t_{3}-t_{2}\right)-f_{2}(\tau) f_{2}\left(t_{3}-t_{2}\right)\right]+\ldots \\
= & 2 f_{1}(\tau)-2 \Re \int_{0}^{\tau} d t_{2} \int_{0}^{t_{2}} d t_{3}\left[f\left(\tau-t_{3}\right) f\left(-t_{2}\right)+f(\tau) f\left(t_{3}-t_{2}\right)\right]+\ldots
\end{aligned}
$$

Upon the change of variables $t_{2} \rightarrow t_{2}-t_{1}, t_{3} \rightarrow t_{3}-t_{1}$ one has for the second and fourth order contribution to $k_{1}(\tau)$ :

$$
\begin{aligned}
& k_{1}^{(2)}\left(t-t_{1}\right)=2 f_{1}\left(t-t_{1}\right) \\
& k_{1}^{(4)}\left(t-t_{1}\right)=-2 \Re \int_{t_{1}}^{t} d t_{2} \int_{t_{1}}^{t_{2}} d t_{3}\left[f\left(t-t_{3}\right) f\left(t_{1}-t_{2}\right)+f\left(t-t_{1}\right) f\left(t_{3}-t_{2}\right)\right] .
\end{aligned}
$$

\section{Appendix B}

Here we derive the contributions up to fourth order to the memory kernel Eq. (36) employing the standard NakajimaZwanzig projection operator technique. Since the initial state of system and bath is of the factorized form Eq. (14) we can employ the standard projection operator

$$
\mathcal{P} w=\operatorname{Tr}_{E}(w) \otimes \rho_{E},
$$

where $w$ is a state of system plus environment and $\rho_{E}$ denotes the vacuum state of the reservoir. This projection operator is the same used to obtain Eq. (34) and for it the initial state Eq. (14) is indeed an eigenoperator. Introducing further the superoperators

$$
\mathcal{L}(t) \rho(t)=-i\left[H_{I}(t), \rho(t)\right]
$$

with $H_{I}(t)$ as in Eq. (6), and

$$
\mathcal{G}\left(t, t_{1}\right)=\mathcal{T} \exp \left(\int_{t_{1}}^{t} \mathrm{~d} s \mathcal{Q L}(s)\right)
$$

where $\mathcal{T}$ denotes time ordering and $\mathcal{Q}=1-\mathcal{P}$, the Nakajima-Zwanzig memory kernel appearing in Eq. (35) is given by

$$
\mathcal{K}_{\mathrm{NZ}}\left(t-t_{1}\right) \rho\left(t_{1}\right)=\operatorname{Tr}_{E}\left(\mathcal{L}(t) \mathcal{G}\left(t, t_{1}\right) \mathcal{Q L}\left(t_{1}\right) \rho\left(t_{1}\right) \otimes \rho_{E}\right) .
$$

Noting that for this model $\mathcal{P} \mathcal{L}\left(t_{1}\right) \ldots \mathcal{L}\left(t_{2 n+1}\right) \mathcal{P}=0$ one has

$$
\begin{aligned}
\mathcal{K}_{\mathrm{NZ}}\left(t-t_{1}\right) \rho\left(t_{1}\right)= & \operatorname{Tr}_{E}\left(\mathcal{L}(t) \mathcal{L}\left(t_{1}\right) \rho\left(t_{1}\right) \otimes \rho_{E}\right) \\
& +\int_{t_{1}}^{t} d t_{2} \int_{t_{1}}^{t_{2}} d t_{3}\left[\operatorname{Tr}_{E}\left(\mathcal{L}(t) \mathcal{L}\left(t_{2}\right) \mathcal{L}\left(t_{3}\right) \mathcal{L}\left(t_{1}\right) \rho\left(t_{1}\right) \otimes \rho_{E}\right)\right. \\
& \left.-\operatorname{Tr}_{E}\left(\mathcal{L}(t) \mathcal{L}\left(t_{2}\right) \mathcal{P} \mathcal{L}\left(t_{3}\right) \mathcal{L}\left(t_{1}\right) \rho\left(t_{1}\right) \otimes \rho_{E}\right)\right]+\ldots
\end{aligned}
$$

Using Eq. (6) and Eq. (16) one readily obtains

$$
\begin{aligned}
\operatorname{Tr}_{E}\left(\mathcal{L}(t) \mathcal{L}\left(t_{1}\right) \rho\left(t_{1}\right) \otimes \rho_{E}\right)= & (-i)^{2}\left[f\left(t-t_{1}\right) \sigma_{+} \sigma_{-} \rho\left(t_{1}\right)-f\left(t_{1}-t\right) \sigma_{-} \rho\left(t_{1}\right) \sigma_{+}\right. \\
& \left.-f\left(t-t_{1}\right) \sigma_{-} \rho\left(t_{1}\right) \sigma_{+}+f\left(t_{1}-t\right) \rho\left(t_{1}\right) \sigma_{+} \sigma_{-}\right]
\end{aligned}
$$


so that the second order contribution is given by

$$
\begin{aligned}
\mathcal{K}_{\mathrm{NZ}}^{(2)}(\tau) \rho= & -i f_{2}(\tau)\left[\sigma_{+} \sigma_{-}, \rho\right] \\
& +2 f_{1}(\tau)\left[\sigma_{-} \rho \sigma_{+}-\frac{1}{2}\left\{\sigma_{+} \sigma_{-}, \rho\right\}\right],
\end{aligned}
$$

which due to Eq (46) confirms the result Eq. (52). Setting

$$
I_{1}\left(t, t_{2}, t_{3}, t_{1}\right) \rho\left(t_{1}\right)=\operatorname{Tr}_{B}\left\{\mathcal{L}(t) \mathcal{L}\left(t_{2}\right) \mathcal{L}\left(t_{3}\right) \mathcal{L}\left(t_{1}\right) \rho\left(t_{1}\right) \otimes \rho_{E}\right\}
$$

and

$$
I_{2}\left(t, t_{2}, t_{3}, t_{1}\right) \rho\left(t_{1}\right)=\operatorname{Tr}_{B}\left\{\mathcal{L}(t) \mathcal{L}\left(t_{2}\right) \mathcal{P} \mathcal{L}\left(t_{3}\right) \mathcal{L}\left(t_{1}\right) \rho\left(t_{1}\right) \otimes \rho_{E}\right\}
$$

a lengthy but straightforward calculation leads to the results

$$
\begin{aligned}
I_{2}\left(t, t_{2}, t_{3}, t_{1}\right) \rho\left(t_{1}\right)= & f\left(t-t_{2}\right) f\left(t_{3}-t_{1}\right) \sigma_{+} \sigma_{-} \rho\left(t_{1}\right)+f\left(t_{2}-t\right) f\left(t_{1}-t_{3}\right) \rho\left(t_{1}\right) \sigma_{+} \sigma_{-} \\
& +2 \Re\left[f\left(t-t_{2}\right) f\left(t_{1}-t_{3}\right)\right] \sigma_{+} \sigma_{-} \rho\left(t_{1}\right) \sigma_{+} \sigma_{-} \\
& -4 f_{1}\left(t-t_{2}\right) f_{1}\left(t_{1}-t_{3}\right) \sigma_{-} \rho\left(t_{1}\right) \sigma_{+}
\end{aligned}
$$

and

$$
\begin{aligned}
I_{1}\left(t, t_{2}, t_{3}, t_{1}\right) \rho\left(t_{1}\right)= & I_{2}\left(t, t_{2}, t_{3}, t_{1}\right) \rho\left(t_{1}\right) \\
& -2 \Re\left[f\left(t-t_{3}\right) f\left(t_{1}-t_{2}\right)+f\left(t-t_{1}\right) f\left(t_{3}-t_{2}\right)\right] \sigma_{-} \rho\left(t_{1}\right) \sigma_{+} \\
& +2 \Re\left[f\left(t-t_{3}\right) f\left(t_{1}-t_{2}\right)+f\left(t-t_{1}\right) f\left(t_{3}-t_{2}\right)\right] \sigma_{+} \sigma_{-} \rho\left(t_{1}\right) \sigma_{+} \sigma_{-} .
\end{aligned}
$$

One thus have for the fourth order expression

$$
\begin{aligned}
\mathcal{K}_{\mathrm{NZ}}^{(4)}\left(t-t_{1}\right) \rho= & -2 \Re \int_{t_{1}}^{t} d t_{2} \int_{t_{1}}^{t_{2}} d t_{3}\left[f\left(t-t_{3}\right) f\left(t_{1}-t_{2}\right)+f\left(t-t_{1}\right) f\left(t_{3}-t_{2}\right)\right] \\
& \times\left[\sigma_{-} \rho \sigma_{+}-\sigma_{+} \sigma_{-} \rho \sigma_{+} \sigma_{-}\right]
\end{aligned}
$$

which according to Eq. (46) confirms Eq. (54).

[1] R. Alicki and K. Lendi, Quantum Dynamical Semigroups and Applications, Vol. 717 of Lecture Notes in Physics, 2nd edn. (Springer, Berlin, 2007)

[2] H.-P. Breuer and F. Petruccione, The Theory of Open Quantum Systems (Oxford University Press, Oxford, 2007)

[3] U. Weiss, Quantum Dissipative Systems, 3rd edn. (World Scientific, Singapore, 2008)

[4] V. Gorini, A. Kossakowski, and E. C. G. Sudarshan, J. Math. Phys. 17, 821 (1976)

[5] G. Lindblad, Comm. Math. Phys. 48, 119 (1976)

[6] H.-P. Breuer, Phys. Rev. A 75, 022103 (2007)

[7] H.-P. Breuer, J. Gemmer, and M. Michel, Phys. Rev. E 73, 016139 (2006)

[8] H.-P. Breuer and B. Vacchini, Phys. Rev. Lett. 101, $140402(2008)$

[9] H.-P. Breuer and B. Vacchini, Phys. Rev. E 79, 041147 (2009)

[10] A. A. Budini, Phys. Rev. E 72, 056106 (2005)

[11] A. A. Budini, Phys. Rev. A 74, 053815 (2006)

[12] A. A. Budini, Phys. Rev. A 69, 042107 (2004)
[13] A. A. Budini and H. Schomerus, J. Phys. A: Math. Gen. 38, 9251 (2005)

[14] D. Chruscinski and A. Kossakowski (2009), eprint arXiv:0912.1259 2

[15] D. Chruscinski, A. Kossakowski, and S. Pascazio (2009), eprint arXiv:0906.5122 2

[16] E. Ferraro, H.-P. Breuer, A. Napoli, M. A. Jivulescu, and A. Messina, Phys. Rev. B 78, 064309 (2008)

[17] A. Kossakowski and R. Rebolledo, Open Syst. Inf. Dyn. 15, 135 (2008)

[18] A. Kossakowski and R. Rebolledo, Open Syst. Inf. Dyn. 16, 259 (2009)

[19] H. Krovi, O. Oreshkov, M. Ryazanov, and D. A. Lidar, Phys. Rev. A 76, 052117 (2007)

[20] J. Piilo, S. Maniscalco, K. Harkonen, and K.-A. Suominen, Phys. Rev. Lett. 100, 180402 (2008)

[21] B. Vacchini, Phys. Rev. A 78, 022112 (2008)

[22] H.-P. Breuer, E.-M. Laine, and J. Piilo, Phys. Rev. Lett. 103, 210401 (2009)

[23] A. Rivas, S. F. Huelga, and M. B. Plenio (2009), eprint arXiv:0911.4270v1 
[24] S. M. Barnett and S. Stenholm, Phys. Rev. A 64, 033808 (2001)

[25] S. Maniscalco, Phys. Rev. A 75, 062103 (2007)

[26] S. Maniscalco and F. Petruccione, Phys. Rev. A 73, 012111 (2006)

[27] A. Shabani and D. A. Lidar, Phys. Rev. A 71, 020101 (2005)

[28] J. Wilkie and Y. M. Wong, J. Phys. A: Math. Gen. 42, 015006 (2009)
[29] S. Daffer, K. Wódkiewicz, J. D. Cresser, and J. K. McIver, Phys. Rev. A 70, 010304 (2004)

[30] V. Weisskopf and E. Wigner, Z. Physik 63, 54 (1930)

[31] J. Fischer and H.-P. Breuer, Phys. Rev. A 76, 052119 (2007)

[32] S. Nakajima, Progr. Theor. Phys. 20, 948 (1958)

[33] R. Zwanzig, J. Chem. Phys. 33, 1338 (1960) 